ANNALS OF

CLINICAL

NEUROPHYSIOLOGY

\title{
Targeting the culprit: vessel wall magnetic resonance imaging for evaluating stroke
}

Seung Min Kim ${ }^{1}$ Sang $\mathrm{Hee} \mathrm{Ha}^{2}$, Hanim $\mathrm{Kwon}^{2}$, Yeon Jung $\mathrm{Kim}^{2}$, Sung Ho $\mathrm{Ahn}^{3}$ Bum Joon $\mathrm{Kim}^{2}$

'Department of Neurology, Veterans Health Service Medical Center, Seoul, Korea

2Department of Neurology, Asan Medical Center, Seoul, Korea

${ }^{3}$ Department of Neurology, Pusan National University Yangsan Hospital, Yangsan, Korea

The pathogenesis of many strokes originates in the vessel wall. Despite this, most traditional imaging focuses on the vascular lumen. Vessel-wall magnetic resonance imaging (VWMRI) is useful for establishing the etiology of intracranial stenosis. It also provides information regarding atherosclerotic plaque composition and thus plaque vulnerability, which is an indication of its potential to cause a stroke. In this review we focus on the characteristics of VWMRI findings in various arteriopathies related to intracranial artery stenosis, and discuss the clinical implications of these findings.

Key words: Vessel-wall magnetic resonance imaging; Ischemic stroke; Intracranial atherosclerosis; Dissection; Moyamoya disease
ORCID

Seung Min Kim

https://orcid.org/0000-0001-5228-6512

Sang Hee Ha

https://orcid.org/0000-0002-2926-2368

Hanim Kwon

https://orcid.org/0000-0003-1340-9529

Yeon Jung Kim

https://orcid.org/0000-0002-6081-890X

Sung Ho Ahn

https://orcid.org/0000-0002-8376-545X

Bum Joon Kim

https://orcid.org/0000-0002-3278-3252

\section{INTRODUCTION}

Ischemic stroke is a cerebrovascular event caused by occlusion or stenosis of the cerebral arteries that supply blood flow to the brain parenchyma. Sudden interruption of this blood flow causes ischemia and subsequent permanent damage to the involved brain tissue, resulting in a focal neurological deficit. More than $40 \%$ of arterial occlusions are attributable to arterial pathology, and predominantly atherosclerosis. ' Various etiologies of arterial stenosis, and stenosis of the intracranial arteries in particular, may cause ischemic stroke.

To date, neuroimaging for stroke has focused on the brain parenchyma, as for other neurological diseases that primarily involve this brain region. Although parenchymal imaging is useful for evaluating the underlying stroke mechanism, predicting the prognosis, and aiding treatment decision-making, it neglects the true culprit of arterial-wall pathology. Various modalities including computed tomography angiography (CTA), magnetic 
resonance angiography (MRA), and digital subtraction angiography (DSA) have been used to locate and measure stenoses in the intra- and extracranial arteries. However, these conventional vascular imaging modalities do not provide adequate information regarding vessel pathology. ${ }^{2}$ Carotid duplex sonography does reveal the vessel-wall pathology of extracranial cervical arteries, ${ }^{3}$ but is of limited use for evaluating intracranial artery stenosis, which is a major cause of stroke in Asian patients.

The use of high-resolution vessel-wall magnetic resonance imaging (MWMRI) to evaluate intracranial arterial-wall pathologies is increasing in many centers. Since treatment decision-making for ischemic stroke depends primarily on the mechanism, it is critical to identify the true culprit. This review focuses on the usefulness of WWMRI for identifying the etiology of arterial stenosis and for evaluating intracranial atherosclerotic stenosis (ICAS). The pitfalls and future directions for the use of WWMRI in the field of stroke care are also discussed.

\section{VWMRI IN ICAS}

\section{Stroke mechanism of ICAS}

It has been demonstrated that the high prevalence of ICAS in Asians may be at least partially explained by vulnerability to certain risk factors, levels of antioxidant enzyme activi$\mathrm{ty}_{1}^{4,5}$ and greater intracranial arterial tortuosity than in other races. ${ }^{6}$ The stroke mechanism also differs according to the location of the atherosclerosis, and most strokes related to extracranial atherosclerosis are caused by artery-to-artery embolism. ICAS is associated with more diverse mechanisms based on the lesion pattern presented on diffusion-weighted imaging: 1) local branch occlusion, 2) artery-to-artery embolism, 3) in situ thrombosis, and 4) hemodynamic. Local branch occlusion is caused by obliteration of the perforator's orifice by the atheroma. Artery-to-artery embolism manifests as infarcts distal to the stenosis in the territory of the relevant artery, usually with small scattered lesions. In situ thrombosis involves infarcts with extensive involvement of most or even all of the diseased arterial territory. With a hemodynamic impairment, the infarcts are located in the border-zone areas associated with perfusion deficits distal to the stenotic or occluded artery. ${ }^{4,7}$ ICAS in the anterior circulation, and especial- ly the distal internal carotid artery (ICA), is more commonly associated with artery-to-artery embolism, whereas local branch occlusion is prevalent in posterior-circulation ICAS, especially in the basilar artery (BA). ${ }^{4}$ In the middle cerebral artery (MCA), the mechanism differs according to the location and severity of the atherosclerotic lesion. In proximal MCA disease, and particularly in the case of proximal MCA occlusion, stroke most commonly results from a combination of mechanisms. This can be explained by plaque rupture with occlusion causing both perforator infarction and embolism. In the case of distal MCA atherosclerotic disease, artery-to-artery embolism is the most common mechanism of stroke because of the location, being associated with turbulence at the distal MCA bifurcation. A longer stenosis is associated with the occurrence of a local branch occlusion. ${ }^{8}$

\section{Imaging techniques in VWMRI}

High-spatial-resolution in VWMRI is essential for characterizing vessel-wall pathologies, enabling visualization of the morphology of both the plaque and its components. In terms of hardware, a magnetic field strength of at least $3 \mathrm{~T}$ is needed to perform WWMRI. ${ }^{9}$ For vessel-wall assessment, a combination of multiple magnetic resonance sequences such as T1-weighted imaging (T1WI), T2-weighted imaging (T2WI), and proton-density (PD) and time of flight (TOF) imaging are used in WWMRI. Multiple tissue-weighted images such as obtained in T1WI, T2WI, and PD imaging are used for better characterization of the individual lesion, whereas TOF imaging is used mainly to characterize luminal abnormalities and locate and assess the site of vessel narrowing. In brain tissue, the signal intensity (SI) on T1WI is highest for lipids, followed in decreasing order by cerebral white matter, cerebral gray matter, and cerebrospinal fluid (CSF). Subacute hemorrhages and high-protein-containing lesions such as mucoceles also have a high SI. On T2Wl, the SI in the brain is highest in the CSF, which has the longest $\mathrm{T} 2$ relaxation time, followed in decreasing order by gray matter and white matter. Brain edema, demyelinating lesions, cerebral infarcts, and tumors also have long T2 relaxation times and thus a high SI on T2WI. Conversely, acute or chronic hemorrhage, calcification, fibrosis, and high-protein-containing lesions have a low SI on T2WI. PD imaging has a better signal-to-noise ratio than T1WI, while contrast enhancement is less evident. ${ }^{9}$ 


\section{Typical findings of VWMRI of ICAS}

Atherosclerosis develops in the inner curvature of the arterial vessel wall, where the shear stress is low. ${ }^{10}$ Atherosclerotic plaques are composed of lipid, fibrous components, cellular material, connective tissue matrix, and calcification. Evaluating these components can aid the diagnosis of vascular pathologies, thus helping to identify "vulnerable" plaques that are at high risk of causing stroke. ${ }^{11}$ ICAS lesions are often multiple and exhibit eccentric thickening with or without a focal enhancement (Fig. 1). Positive vessel-wall remodeling can be also observed in some cases. ${ }^{12}$ Symptomatic plaques are associated with more positive vessel-wall remodeling, intraplaque hemorrhage, and increased vessel-wall enhancement. The aspects of WWMRI findings used to differentiate ICAS from other vascular pathologies and to identify vulnerable plaques are described below.

\section{Plaque enhancement}

Plaque enhancement on contrast-enhanced T1WI is the most common and reliable finding. Plaque enhancement is caused by neovascularization or increased endothelial permeability by inflammation. However, normal periarterial enhancement in the meninges or periarterial veins can be confused with true vascular-wall enhancement. Therefore, performing three-dimensional WWMRI with multiple imaging planes, such as perpendicular to the arterial longitudinal axis, can reduce misreading. In the enhanced plaque, several components with different enhancements may be identified. The plaque layer adjacent to the vascular lumen ap- pears hyperintense on $\mathrm{T} 2 \mathrm{Wl}$ and may show enhancement. In contrast, a hypointense and nonenhanced layer in the adjacent area is often observed on T2WI. A third, thin layer with enhancement is often observed in the periphery. These layers observed on carotid WWMRI are correlated with findings obtained from carotid endarterectomy specimens. The enhanced layer immediately adjacent to the lumen is the fibrous cap, the outer nonenhanced layer indicates the lipid core, and the outer peripheral thin rim of enhancement represents an increased area of vasa vasorum in the arterial adventitia (Table 1). ${ }^{13}$ However, not all intracranial atherosclerotic plaques contain these layers or show enhancement.

Plaque enhancement is associated not only with recent ischemic stroke, but also with the risk of future stroke recurrence. ${ }^{14}$ Strong enhancement is associated with a short time interval from ischemic stroke onset, and suggests severe inflammation. ${ }^{15}$ A postmortem study of MCA plaques found a correlation between neovascularity and the presence of an ipsilateral infarction. ${ }^{16}$ Symptomatic plaques exhibit contrast enhancement more often than do asymptomatic plaques. The degree of contrast enhancement gradually decreases over time, ${ }^{15}$ but persistence of plaque enhancement at follow-up imaging is thought to be a predictor of the risk of future stroke recurrence. ${ }^{14}$ However, these findings should be interpreted with caution, since $23 \%$ of nonculprit plaques were also enhanced.

Vessel-wall enhancement can also appear in other generalized inflammatory diseases; therefore, the presence of arterial-wall enhancement alone cannot exclude other diseases.
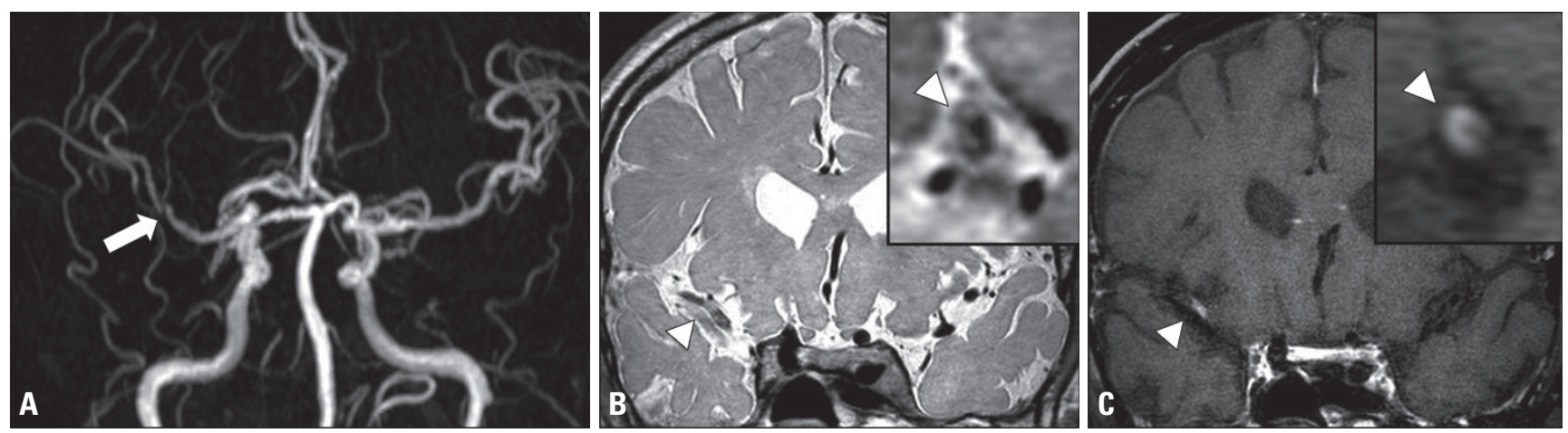

Fig. 1. Vessel-wall magnetic resonance imaging findings in a 68-year-old male with intracranial atherosclerosis. (A) Time of flight magnetic resonance angiography showing severe stenosis in the right middle cerebral artery (arrow) with few branch vessels. (B) Proton density imaging showing eccentric vessel-wall thickening and atherosclerotic plaques (arrowhead). (C) Contrast-enhanced T1-weighted imaging showing enhancement in eccentric atherosclerotic plaques (arrowheads). 
Table 1. Signal intensity of atherosclerotic plaque components on VWMRI

\begin{tabular}{lcccc}
\hline Plaque component & T1WI & T2WI & PD imaging & Contrast-enhanced T1WI \\
\hline Fibrotic tissue & Iso/hyper & Iso/hyper & Iso/hyper & Yes \\
Lipid & Iso/hyper & Hypo & Iso/hyper & No \\
Calcification & Hypo & Hypo & & Hypo \\
Hemorrhage $^{\text {a }}$ & & & No \\
Acute (<1 week) & Hyper & Iso/hypo & Hyper & No \\
Subacute (1-6 weeks) & Hyper & Hyper & Hypo & No \\
Chronic (>6 weeks) & Hypo & Hypo & Hypo & Ho \\
\hline
\end{tabular}

Signal intensity is compared with that of adjacent muscles.

VWMRI, vessel-wall magnetic resonance imaging; T1Wl, T1-weighted imaging; T2Wl, T2-weighted imaging; PD, proton density; Iso, isointense; hyper, hyperintense; hypo, hypointense.

aTime to observation.

Given the relative lack of vasa vasorum in ICAS compared with the coronary and extracranial carotid arteries, plaque enhancement is a more useful imaging finding in ICAS, being more suggestive of neovascularization or inflammation than vasa-vasorum-induced enhancement. ${ }^{17}$ The reduced vasa vasorum in the intracranial arteries is attributable to the surrounding CSF, which can supply nutrients via diffusion.

\section{Intraplaque hemorrhage}

Intraplaque hemorrhage is a common feature of atherosclerosis and its occurrence is strongly correlated with atherosclerotic plaque progression, instability, and rupture. ${ }^{18}$ The SI of the intraplaque hemorrhage depends on both oxygenation status and hemoglobin structure. In general, fresh hemorrhage observed within 1 week of onset corresponds to intracellular methemoglobin and exhibits high SI on T1WI and TOF images, and iso- or low SI on T2WI and PD images. Recent intraplaque hemorrhage observed 1-6 weeks after onset is associated with extracellular methemoglobin, exhibiting high $\mathrm{SI}$ in all imaging sequences. Chronic hemorrhage observed more than 6 weeks after onset is associated with extracellular hemosiderin and exhibits low SI in all four sequences (Table 1). ${ }^{19}$ Cervical carotid atherosclerotic plaques with high SI on T1WI that is greater than 1.5 times brighter than the SI of adjacent muscles can confirm the presence of recent hemorrhage. This is the same as seen in MRI findings of intracerebral hemorrhage. Both postmortem and WWMRI studies of MCA stenosis confirm that intraplaque hemorrhage is related to the occurrence of ischemic stroke, sug- gesting a vulnerable plaque. ${ }^{16}$

\section{Vessel-wall thickening}

The morphological features of concentric and eccentric vessel-wall thickening have been described that distinguish plaques from other vasculopathies. Atherosclerotic plaques on WWMRI of ICAS differ from other vascular pathologies by the presence of asymmetrically thickened walls (eccentricity). ${ }^{12}$ Quantitative measurement and analysis of the plaque shape can be a more reliable method of assessment than visual analysis in this case. An eccentric plaque is defined by an eccentricity index ([maximum thickness-minimum thickness]/ maximum thickness) of $\geq 0.5$. Although early studies found that eccentric vessel-wall thickening was a typical characteristic of intracranial plaques, more recent reports describe ICAS with concentric vessel-wall thickening. ${ }^{12}$ A VWMRI study of the BA revealed that plaques first develop at the inner curvature of vertebrobasilar junction (posterior wall) and extend to the lateral wall, showing a more concentric nature. $^{20}$

Recent histopathological studies have shown that atherosclerotic plaques can cause both eccentric and concentric vessel-wall thickening. One study found that MCA plaques were more eccentric (69\%), whereas plaques in the vertebral artery (VA) (75\%) and BA (62\%) were more concentric. ${ }^{21}$ Another study found that concentric plaques were more common than eccentric plaques in the MCA $(63.9 \%$ vs. $26.1 \%$, respectively). ${ }^{22}$ Therefore, the specificity of plaque eccentricity is not high, and intracranial plaques can manifest as 
both eccentric and concentric vessel-wall thickening. Most studies have found symptomatic intracranial plaques to be thicker than asymptomatic plaques, but there is one report of no significant relationship between plaque thickness and vulnerability. ${ }^{13}$ For the MCA, the plaque surface of symptomatic stenoses is more irregular than for their asymptomatic counterparts. ${ }^{23}$

\section{Vessel-wall remodeling}

Atherosclerotic plaques often cause outward bulging of the outer surface of the artery. This compensates for the arterial stenosis caused by atherosclerotic vessel-wall thickening, and subsequently preserves the lumen size and hence the blood flow. This outward vascular remodeling is called "positive remodeling," and it occurs more frequently in the posterior circulation because of the difference in sympathetic innervation and flow dynamics. ${ }^{24}$ This positive remodeling of the intracranial arteries results in less luminal narrowing than negative (inward) remodeling, but is more often associated with intraplaque hemorrhage and severe inflammation. ${ }^{25}$ In addition, transcranial Doppler sonography has revealed that positive remodeling is more commonly associated with microembolic signals than negative remodeling. ${ }^{26}$ Therefore, a plaque with positive remodeling indicates a high-risk or vulnerable plaque. ${ }^{19}$ Positive remodeling is defined as a WWMRI remodeling index (maximum outer wall area/[proximal normal arterial area + distal normal arterial area/2]) of $\geq 1.0$.

\section{Other components}

The appearance of lipid cores, fibrous components, and calcification in intracranial plaques is not sufficiently differentiated on WWMRI. The lipid core is isointense to hyperintense on T1WI and PD imaging, hypointense to isointense area on T2WI (Table 1), and exhibits less enhancement compared with fibrous tissue on contrast-enhanced T1WI. However, the $\mathrm{SI}$ of the lipid core may vary, probably due to differences in the amount of liquid components and the echo times used in T2WI. ${ }^{27}$ The lipid core in the liquid phase flows out from the ruptured site of the fibrous cap, causing a cerebrovascular embolism. Conversely, organized lipid cores are firmer, more stable, and more resistant to plaque rupture. Fibrous components are isointense or hyperintense compared with adjacent muscles on T1WI, T2WI, and PD imaging, but isointense on TOF imaging. Fibrous tissue shows enhancement on contrast-enhanced T1WI, whereas the lipid-rich necrotic cores do not. Calcification is characterized by marked dark SI on T1WI, T2WI, and PD and TOF imaging. Table 1 summarizes the SIs of the various plaque components on WWMRI.

\section{VWMRI IN NONATHEROSCLEROTIC DIS- EASE}

\section{Moyamoya disease}

Moyamoya disease (MMD) is an idiopathic, progressive cerebrovascular disease that is characterized by stenosis or occlusion of the distal portion of the ICA and the proximal segments of the MCA and anterior cerebral artery. ${ }^{28}$ The progressive nature of this disease results in the formation of a fine meshwork of collateral vessels, named Moyamoya vessels. Although these vascular changes generally occur bilaterally, unilateral vascular involvement is often observed in adult-onset MMD. ${ }^{29}$ Changes in the inner diameter of the vessel are not apparent in the very early stages of MMD.

The gold-standard diagnostic tool for MMD is DSA. However, DSA is relatively invasive and may cause procedure-related complications. Conventional MRA or CTA are noninvasive, but have poor sensitivity in detecting basal collateral vessels. ${ }^{30}$ WWMRI is a noninvasive technique that has the advantage of allowing the evaluation of vascular-wall pathology. Patients with ICAS often present with positive remodeling of the stenotic lesion, while their counterparts with MMD usually present with smaller outer diameters and negative remodeling qqqon WWMRI (Table 2). ${ }^{31}$ Histopathological studies have demonstrated that the vessel outer diameter is smaller in patients with MMD than in their nonMMD counterparts. ${ }^{32}$ Several WWMRI studies have shown that the affected vessels in MMD undergo negative remodeling, resulting in a reduction in the ratio of the outer-vessel-wall boundary area at the lesion site to that at the reference site. $^{31}$ In the lesion at the site of the stenosis, atherosclerotic plaques usually present with an eccentric pattern and a heterogeneous SI, while in MMD these plaques often exhibit a concentric pattern and a homogeneous SI (Fig. 2). ${ }^{33}$ The histopathological findings of vessels in MMD, which are reflected on WWMRI, are intimal thickening with increased smooth-muscle cells or endothelium. ${ }^{34}$ Whether or not the vascular stenosis is enhanced remains to be established, 
Table 2. Common imaging characteristics of intracranial artery disease on VWMRI

\begin{tabular}{|c|c|c|c|c|c|}
\hline & $\begin{array}{c}\text { Intracranial } \\
\text { atherosclerosis }\end{array}$ & Moyamoya disease & Arterial dissection & RCVS & CNS vasculitis \\
\hline Vessel-wall thickening & $\begin{array}{l}\text { Eccentric (sometimes } \\
\text { concentric) }\end{array}$ & Concentric & Eccentric & Concentric & $\begin{array}{l}\text { Concentric (sometimes } \\
\text { eccentric) }\end{array}$ \\
\hline Location & Any artery & $\begin{array}{l}\text { Usually anterior circulation } \\
\text { (distal ICA, proximal MCA, } \\
\text { and ACA) }\end{array}$ & $\begin{array}{l}\text { Any artery (distal ICA and } \\
\text { VA) }\end{array}$ & $\begin{array}{l}\text { Medium to small } \\
\text { sized arteries }\end{array}$ & $\begin{array}{l}\text { Medium to small sized } \\
\text { arteries (more wide- } \\
\text { spread) }\end{array}$ \\
\hline Enhancement & $\begin{array}{l}\text { Eccentric } \\
\text { Dependent on stage } \\
\qquad(+++\rightarrow+)\end{array}$ & $+/-$ & Often present & $+/-$ & $\begin{array}{l}\text { Concentric } \\
\text { Dependent on stage } \\
(+++\rightarrow-)\end{array}$ \\
\hline Outer diameter & $\begin{array}{l}\text { Positive or negative } \\
\text { remodeling }\end{array}$ & Negative remodeling & $\begin{array}{l}\text { Positive (subadventitial } \\
\text { hematoma) or negative } \\
\text { remodeling (subintimal } \\
\text { hematoma) }\end{array}$ & & \\
\hline Others & $\begin{array}{l}\text { Intraplaque } \\
\text { hemorrhage }\end{array}$ & $\begin{array}{l}\text { Moyamoya vessels (basal } \\
\text { collateral vessels) }\end{array}$ & $\begin{array}{l}\text { Intimal flap, double lumen, } \\
\text { intramural hematoma, } \\
\text { or aneurysmal dilatation }\end{array}$ & $\begin{array}{l}\text { Multifocal segmen- } \\
\text { tal vasoconstric- } \\
\text { tion }\end{array}$ & \\
\hline Clinical consideration & Stroke risk factors & Progressive, family history & Trauma & Reversibility & $\begin{array}{l}\text { Effect of steroid therapy, } \\
\text { systemic disease }\end{array}$ \\
\hline
\end{tabular}

VWMRI, vessel-wall magnetic resonance imaging; RCVS, reversible vasoconstriction syndrome; CNS, central nervous system; ICA, internal carotid artery; MCA, middle cerebral artery; ACA, anterior cerebral artery; VA, vertebral artery.
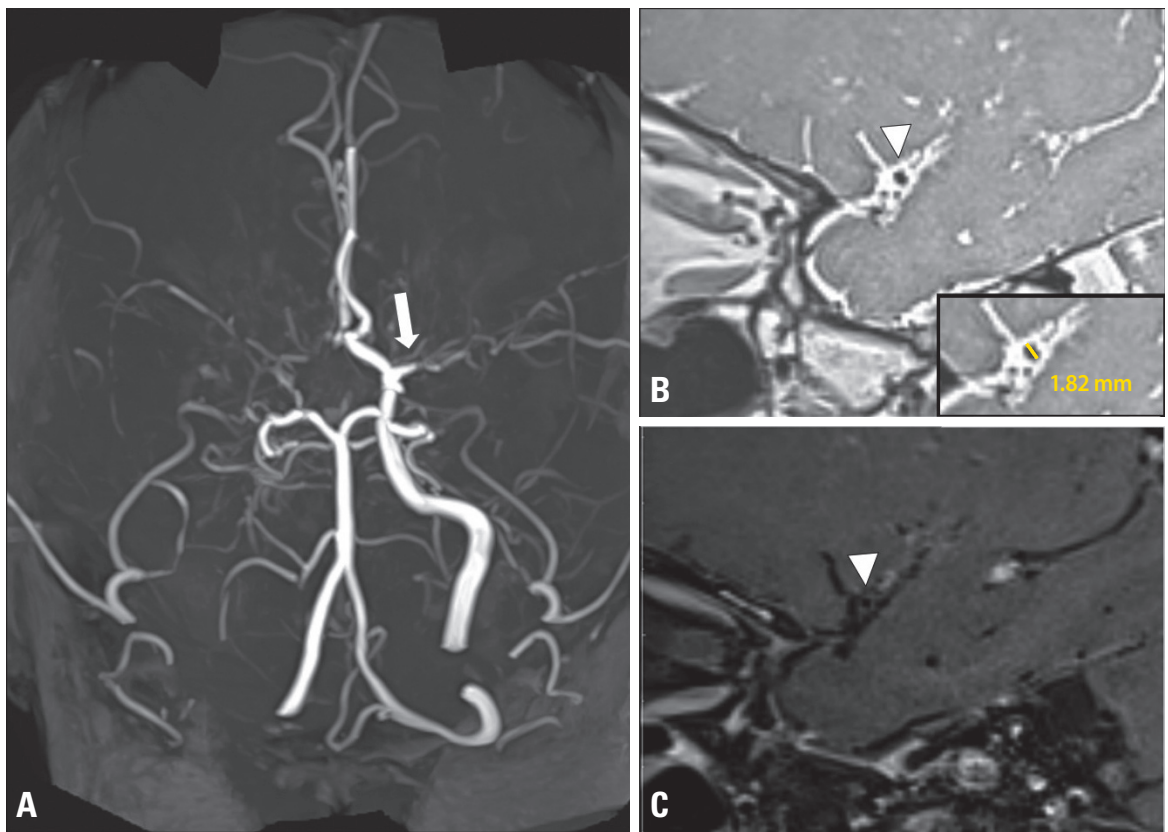

Fig. 2. Vessel-wall magnetic resonance imaging findings in a 62-year-old female with Moyamoya disease. (A) Time of flight magnetic resonance angiography demonstrating stenosis of the right distal internal carotid artery, anterior cerebral artery, and bilateral middle cerebral arteries (arrow). (B) Proton density imaging showing concentric narrowing, decreased vessel-wall diameter, and negative remodeling (arrowhead). (C) Contrast-enhanced T1-weighted imaging showing minimal concentric enhancement (arrowhead). 
with findings varying between studies. However, when this enhancement is observed in MMD, focal enhancement is not as common as in ICAS, ${ }^{33}$ and reportedly exhibits a relatively concentric pattern. ${ }^{28}$ The location of the vascular stenosis is also a diagnostic clue in MMD, with steno-occlusion of the affected vessel usually beginning in the anterior circulation and then proceeding to the posterior circulation. ${ }^{31,33}$

\section{Dissection}

Intracranial arterial dissection (IAD) is an important cause of stroke, especially for young patients, accounting for up to $20 \%$ of cases in this population. Whereas extracranial artery dissection (EAD) predominates (90\%) in Western countries, IAD is more frequent (67-90\%) in Asian countries, ${ }^{35,36}$ where the VA is the most common dissection location. ${ }^{35-37}$ Dissection manifests in various ways, including ischemic symptoms and subarachnoid hemorrhage (SAH). Ischemic stroke and transient ischemic attack present in about 60\% of patients with EAD and in 30-78\% of those with IAD. ${ }^{36}$ IAD presents as SAH in 20-60\% of patients and accounts for 3-5\% of all SAHs. ${ }^{36}$ Clinical outcomes after dissection are generally favorable, with a particularly low annual recurrent ischemic stroke rate of $0.3-2.4 \%$ for $E A D$, and a low rate of $2-14 \%$ for IAD compared with 19\% for ICAS. ${ }^{36}$

VWMRI is useful for diagnosing arterial dissection even if conventional luminal angiography does not reveal the typical features. The characteristic features of arterial dissection are an intimal flap, double lumen, intramural hematoma, aneurysmal dilatation, tapered stenosis, and occlusions (Table 2). Among these findings, the intimal flap and double lumen are pathognomonic features according to the criteria of the
Strategies against Stroke Study for Young Adults in Japan. ${ }^{38}$ However, the detection rate of these signs on DSA is reportedly less than 10\%; the detection rate of an intimal flap on WWMRI has been reported to range from $42 \%$ to $91 \%{ }^{36}$ The presence of an intimal flap indicates tearing of the intima and is thus regarded as direct evidence of arterial dissection. This flap appears as a hyperintense curvilinear line that is separated from the vessel lumen on T2WI (Fig. 3). A false lumen is created when blood dissects through the arterial wall and re-enters the vessel lumen. Together with the true lumen, this feature is labeled the double-lumen sign.

The incidence of intramural hematoma, one of the most common findings of IAD, ranges from $61 \%$ to as high as $100 \% .{ }^{35,39}$ It may occur as a result of either direct intimal tear with penetration of the blood (subintimal hematoma) or extravasation from the vasa vasorum (subadventitial hematoma). Intramural hematomas are typically described as crescent-shaped thickenings of the arterial wall, which compress the lumen to cause steno-occlusion or dilatation of its external diameter, leading to aneurysmal change. The shape of the hematoma is associated with the presence of ischemic symptoms. ${ }^{40}$ Intramural hematomas have high $\mathrm{SI}$ on T1WI due to an increased methemoglobin component during the subacute stage (2 days to 2 months). However, the $\mathrm{SI}$ changes according to the age of the hematoma: during the acute stage (1-2 days) they are hypointense on T1WI and T2WI, becoming isointense after 2 months, rendering their detection difficult. ${ }^{39,41}$ Susceptibility-weighted imaging is also used for the detection of intramural hematomas. Differentiating between an intramural hematoma and hemorrhagic intraplaque rupture is sometimes difficult. Sub-
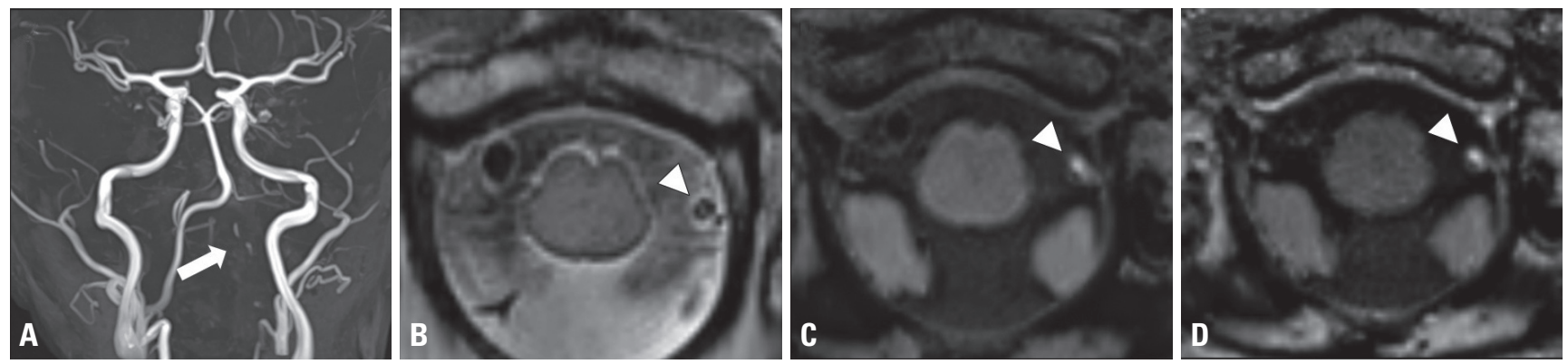

Fig. 3. Vessel-wall magnetic resonance imaging findings in a 33-year-old female with vertebral artery dissection. (A) Time of flight magnetic resonance angiography showing occlusion of the left V3-V4 segment of the vertebral artery (arrow). (B) On proton density imaging, an intimal flap was observed as a line in the lumen (arrowhead). (C) T1-weighted imaging (T1WI) showing a hyperintense, crescent-shaped intramural hematoma (arrowhead). (D) Contrast-enhanced T1WI showing eccentric enhancement (arrowhead). 
intimal hematoma causing stenosis is difficult to distinguish from intraplaque hemorrhage with negative remodeling, and subadventitial hematoma with aneurysmal dilatation is likely to be confused with an intraplaque hemorrhage with positive remodeling. Therefore, clinical information such as cardiovascular risk factors and age, together with other radiological findings is required to distinguish them. ${ }^{35,36}$

While the pathogenesis of vessel-wall enhancement is not yet fully understood, it is thought to be associated with inflammation, enhancement of the vasa vasorum, slow blood flow through a false lumen, or thrombosis formation from the intramural hematoma. ${ }^{35}$ The findings of several studies suggest that the enhanced portion of an intramural hematoma represents intramural thrombus formation and that this is associated with increased risk of ischemic stroke. ${ }^{42}$ The enhancement pattern of a dissection tends to be eccentric and sometimes extends beyond the extent of the intimal flap and/or hematoma, suggesting that the affected area would be larger than just the site of the dissection.

Aneurysmal dilatation is caused by the accumulation of blood through the dissection site, which subsequently cleaves vessel layers, especially between the media and adventitia. A WWMRI study found that aneurysmal dilatation was the most common finding of IAD, accounting for $67 \%$ of all cases. ${ }^{43}$ Rupture of an intracranial dissecting aneurysm leads to SAH. The intradural segment of the VA is the site of SAHs in more than $50 \%$ of cases. ${ }^{43}$ Although not specific signs, the presence of a fusiform or irregularly shaped aneurysm and location in a nonbranching arterial site with segmental stenosis can raise suspicion of dissection.

In the chronic stage, an intimal flap, double-lumen sign, aneurysmal dilatation, and intramural hematoma tend to disappear. However, some cases demonstrate persistent mild enhancement with focal vessel-wall thickening in the chronic stage, even if the stenosis has completely resolved. It is our opinion that these changes may be attributable to compensatory intimal hyperplasia. Stenosis resolution was shown to occur in up to $90 \%$ of EAD patients, ${ }^{44}$ while the rate of improvement of stenosis among IAD patients varied markedly between studies, from $20 \%$ to $84 \%{ }^{35,36}$ Improvement in stenosis usually occurs within 3-6 months of the dissection event and is rarely observed after 6 months. ${ }^{41,44}$

\section{Reversible cerebral vasoconstriction syndrome}

Reversible cerebral vasoconstriction syndrome (RCVS) is a clinical and radiological syndrome that manifests with the primary clinical features of sudden onset of severe headache and diffuse segmental vasoconstriction of the cerebral arteries that resolves within 3 months. ${ }^{45}$ However, RCVS is not a single disease entity, and its diagnosis can be challenging due to its symptoms and signs overlapping with those of a range of other central nervous system (CNS) diseases that are accompanied by the sudden onset of severe headache. Multifocal segmental cerebral artery vasoconstriction demonstrated on either DSA or indirectly on CTA/MRA that normalizes within 12 weeks after onset is a characteristic finding of RCVS.

WWMRI can differentiate the distinct characteristics of arterial-wall thickening and arterial-wall enhancement, which can be helpful for the timely and accurate diagnosis and management of RCVS and the overlapping non-RCVS disease entity. ${ }^{46,47}$ Recent studies have identified characteristics of arterial-wall thickening and arterial-wall enhancement in RCVS that are distinct from those of CNS vasculitis and $\mathrm{SAH}^{48}$ Both vasculitis and RCVS exhibit arterial-wall thickening, but RCVS has no or mild arterial-wall enhancement compared with vasculitis (Table 2). When aneurysmal-wall enhancement is observed in vasospasm associated with aneurysmal SAH, it is helpful to rule out RCVS because it indicates inflammation and possible recent rupture. Distinguishing between RCVS and other non-RCVS emergencies is critical due to the significant differences in their treatments and clinical outcomes. The course of RCVS is rather benign, with spontaneous resolution occurring and few complications compared with the other, non-RCVS emergencies; for example, CNS vasculitis requiring immunotherapy and $\mathrm{SAH}$ requiring intensive care management and surgical/ interventional treatment, usually with a poor outcome. Furthermore, serial monitoring based on noninvasive WWMRI can be useful to differentiate the following dynamic pattern: delay in the appearance of RCVS vasoconstriction that may mimic the typical time course of arterial vasospasm, while hyperacute vasospasm may occasionally be associated with aneurysmal SAH.

\section{Vasculitis}

CNS vasculitis is a rare disease that can be classified into 
primary CNS angiitis and secondary CNS vasculitis due to systemic inflammation or infectious conditions. ${ }^{49}$ Pathologically, vasculitis is characterized by luminal narrowing, wall disruption, ischemia, and hemorrhage due to inflammation of the vessel wall. These characteristics mean that VWMRI, which can visualize vessel-wall inflammation, may be helpful for diagnosing vasculitis. Although visualizing the walls of small intracranial vessels is difficult with the current spatial resolution of VWMRI, this method is useful when the CNS vasculitis involves larger intracranial arteries. CNS vasculitis is typically characterized on WWMRI by smooth, homogeneous, concentric vessel-wall thickening and contrast enhancement, more predominantly in small to medium-sized arteries (Table 2, Fig. 4). This differs from atherosclerotic plaques, in which eccentric and irregular vessel-wall abnormalities occur predominantly in the more proximal intracranial arteries. However, since atherosclerotic plaques sometimes show concentric vessel-wall thickening, vasculitis can also show eccentric vessel-wall thickening and enhancement.

The likely mechanisms underlying arterial-wall enhancement in CNS vasculitis are increased permeability of the endothelium with contrast leakage from the lumen into the arterial wall, and vasa-vasorum-related contrast leakage. Arterial involvement is more common where there are single lesions in multiple different arteries than where there are multiple lesions in a single artery. ${ }^{50}$ Thus, vasculitis should be suspected when multiple ischemic strokes occur with various ages at onset in different vascular territories. ${ }^{49}$ The presence or severity of vessel-wall enhancement is not helpful in the differential diagnosis of vasculitis etiology, whereas the distribution of a vascular involvement pattern is. For
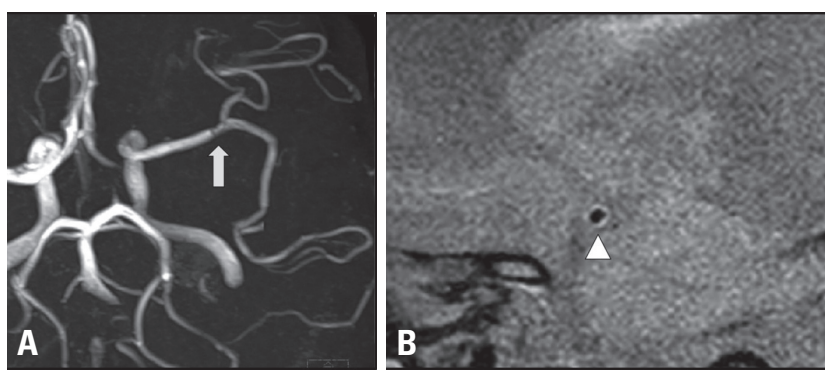

Fig. 4. Vessel-wall magnetic resonance imaging findings in a 30-yearold male with vasculitis. (A) Time of flight magnetic resonance angiography showing multifocal stenosis of the left middle cerebral artery (arrow). (B) Contrast-enhanced proton density imaging indicating a concentric stenosis with diffuse circumferential enhancement (arrowhead). example, temporal arteritis can be suspected if vasculitis affects the superficial temporal artery and there is concurrent involvement of the intradural ICA or VA. Primary CNS angiitis may be suspected in cases involving medium-sized vessels, such as those distal to the MCA bifurcation, or the anterior or posterior communicating arteries. ${ }^{50}$ In intracranial artery vasculitis, clinical disease activity and WWMRI findings are not always correlated. However, certain treatments affect WWMRI findings, such as the diagnostic sensitivity of WWMRI decreasing when corticosteroid is administered in patients with temporal arteritis. The different imaging characteristics in intracranial artery diseases on WWMRl are presented in Table 2.

\section{Pitfalls of VWMRI}

VWMRI is clinically useful, but appropriate imaging techniques and proper interpretation are important for an accurate diagnosis. It is also important to be aware of the potential pitfalls and limitations for the proper application of VWMRI in clinical settings. The clinical implications of an ICAS plaque are understood based on the results of carotid plaque studies, but it is unclear whether the extents of the carotid and intracranial plaques are actually similar. Few studies have correlated histopathological and WWMRI findings. Moreover, it is difficult to achieve adequate spatial resolution with WWMRI of ICAS, and caution is needed when interpreting the findings relative to those obtained for carotid plaques. Therefore, certain factors should be considered in the interpretation. First, slow vascular flow can cause the loss of flow voids and mimic vessel-wall enhancement. ${ }^{9,51}$ The WWMRI technique relies on blood flow for blood-signal suppression. Since the blood in a vessel exhibits laminar flow, the velocity is lower closer to the vessel wall. Therefore, the area adjacent to the vessel wall may exhibit incomplete signal suppression, giving the impression of vessel-wall thickening or enhancement. Artifacts are also likely to occur as a result of recirculating or slow blood flow due to an aneurysm or dilated artery, and retrograde filling through a leptomeningeal collateral due to proximal arterial occlusion.

Second, the blood supply to the vasa vasorum of the intracranial arteries may appear like concentric arterial-wall thickening and concentric enhancement, potentially mimicking vasculitis. ${ }^{9,51}$ The vessel wall of the extracranial arteries receives its blood supply through the vasa vasorum, whereas the intracranial arteries rely less on the vasa vasorum, which 
is smaller than in extracranial arteries, due to the influence of the adjacent CSF. Aging and atherosclerotic risk factors cause the extracranial vasa vasorum to extend to the proximal intracranial segment of the ICA or VA, and the vasa vasorum develops in the intracranial arteries. In the presence of ICAS, the vasa vasorum can develop more distant from the extracranial vasa vasorum, ${ }^{17}$ causing concentric arterial-wall thickening and enhancement, mimicking vasculitis.

Third, in the case of mechanical thrombectomy, concentric intracranial arterial-wall thickening and enhancement may be seen due to mechanical injury of the arterial wall. Thus, post-thrombectomy enhancement can be misdiagnosed as CNS vasculitis. ${ }^{52}$ Finally, venous enhancement adjacent to arteries can also mimic vessel-wall enhancement. ${ }^{9,51}$ This problem can be avoided by performing VWMRI with multiple planes and comparing the images with MRA findings.

\section{CONCLUSION}

WWMRI enables direct visualization of the vessel-wall pathology causing arterial stenosis, and is therefore useful for determining the etiology of that stenosis. Furthermore, WWMRI also provides information regarding atherosclerotic plaque components, which reflect its vulnerability. The mechanism underlying stroke and the risk of stroke recurrence are associated with plaque vulnerability. Despite the existence of several pitfalls, WWMRI is useful for investigating the true culprit underlying stroke (arterial stenosis), especially in patients with intracranial arterial pathology.

\section{Funding source}

This research was supported by the Brain Convergence Research Program of the National Research Foundation (NRF) funded by the Korean government (MSIT; No. 2020M3E5D2A01084576).

\section{Conflict of interest}

The authors declare no conflicts of interest relevant to this article.

\section{REFERENCES}

1. Kim BJ, Kim JS. Ischemic stroke subtype classification: an asian viewpoint. J Stroke 2014;16:8-17.

2. Kim BJ, Kang HG, Kim HJ, Ahn SH, Kim NY, Warach S, et al. Magnetic resonance imaging in acute ischemic stroke treatment. J Stroke 2014;16:131-145.

3. Kim SM, Kim YJ, Kim K, Kim BJ. Usefulness of carotid ultrasonography and treatment of carotid disease. J Korean Med Assoc 2020;63:342-353.

4. Kim JS, Nah HW, Park SM, Kim SK, Cho KH, Lee J, et al. Risk factors and stroke mechanisms in atherosclerotic stroke: intracranial compared with extracranial and anterior compared with posterior circulation disease. Stroke 2012;43:3313-3318.

5. Kim JS, Bonovich D. Research on intracranial atherosclerosis from the East and west: why are the results different? J Stroke 2014; 16:105-113.

6. Kim BJ, Lee KM, Lee SH, Kim HG, Kim EJ, Heo SH, et al. Ethnic differences in intracranial artery tortuosity: a possible reason for different locations of cerebral atherosclerosis. J Stroke 2018;20:140141.

7. Kim YS, Kim BJ, Noh KC, Lee KM, Heo SH, Choi HY, et al. Distal versus proximal middle cerebral artery occlusion: different mechanisms. Cerebrovasc Dis 2019;47:238-244.

8. Ha SH, Chang JY, Lee SH, Lee KM, Heo SH, Chang DI, et al. Mechanism of stroke according to the severity and location of atherosclerotic middle cerebral artery disease. J Stroke Cerebrovasc Dis 2021;30:105503

9. Mandell DM, Mossa-Basha M, Qiao Y, Hess CP, Hui F, Matouk C, et al. Intracranial vessel wall MRl: Principles and Expert Consensus Recommendations of the American Society of Neuroradiology. AJNR Am J Neuroradiol 2017;38:218-229.

10. Kim BJ, Yoon Y, Lee DH, Kang DW, Kwon SU, Kim JS. The shape of middle cerebral artery and plaque location: high-resolution MRI finding. Int J Stroke 2015;10:856-860.

11. Fuster V, Moreno PR, Fayad ZA, Corti R, Badimon JJ. Atherothrombosis and high-risk plaque: part I: evolving concepts. J Am Coll Cardiol 2005;46:937-954.

12. Dieleman N, Yang W, Abrigo JM, Chu WC, van der Kolk AG, Siero $J C$, et al. Magnetic resonance imaging of plaque morphology, burden, and distribution in patients with symptomatic middle cerebral artery stenosis. Stroke 2016;47:1797-1802.

13. Xu WH, Li ML, Gao S, Ni J, Zhou LX, Yao M, et al. In vivo high-resolution MR imaging of symptomatic and asymptomatic mid- 
dle cerebral artery atherosclerotic stenosis. Atherosclerosis 2010;212:507-511.

14. Kim JM, Jung KH, Sohn CH, Moon J, Shin JH, Park J, et al. Intracranial plaque enhancement from high resolution vessel wall magnetic resonance imaging predicts stroke recurrence. Int J Stroke 2016;11:171-179.

15. Vakil P, Vranic J, Hurley MC, Bernstein RA, Korutz AW, Habib A, et al. T1 gadolinium enhancement of intracranial atherosclerotic plaques associated with symptomatic ischemic presentations. AJNR Am J Neuroradiol 2013;34:2252-2258.

16. Chen XY, Wong KS, Lam WW, Zhao HL, Ng HK. Middle cerebral artery atherosclerosis: histological comparison between plaques associated with and not associated with infarct in a postmortem study. Cerebrovasc Dis 2008;25:74-80.

17. Portanova A, Hakakian N, Mikulis DJ, Virmani R, Abdalla WM, Wasserman BA. Intracranial vasa vasorum: insights and implications for imaging. Radiology 2013;267:667-679.

18. Parma L, Baganha F, Quax PHA, de Vries MR. Plaque angiogenesis and intraplaque hemorrhage in atherosclerosis. Eur J Pharmacol 2017;816:107-115.

19. Chu B, Kampschulte A, Ferguson MS, Kerwin WS, Yarnykh VL, $\mathrm{O}$ Brien KD, et al. Hemorrhage in the atherosclerotic carotid plaque: a high-resolution MRI study. Stroke 2004;35:1079-1084.

20. Kim BJ, Kim HY, Jho W, Kim YS, Koh SH, Heo SH, et al. Asymptomatic basilar artery plaque distribution and vascular geometry. J Atheroscler Thromb 2019;26:1007-1014.

21. Yang WJ, Fisher M, Zheng L, Niu CB, Paganini-Hill A, Zhao HL, et al. Histological characteristics of intracranial atherosclerosis in a Chinese population: a postmortem study. Front Neurol 2017:8:488

22. Yang WJ, Chen XY, Zhao HL, Niu CB, Xu Y, Wong KS, et al. In vitro assessment of histology verified intracranial atherosclerotic disease by $1.5 T$ magnetic resonance imaging: concentric or eccentric? Stroke 2016:47:527-530

23. Chung GH, Kwak HS, Hwang SB, Jin GY. High resolution MR imaging in patients with symptomatic middle cerebral artery stenosis. Eur J Radiol 2012:81:4069-4074.

24. Qiao Y, Anwar Z, Intrapiromkul J, Liu L, Zeiler SR, Leigh R, et al. Patterns and implications of intracranial arterial remodeling in stroke patients. Stroke 2016;47:434-440.

25. Pasterkamp G, Schoneveld AH, van der Wal AC, Hijnen DJ, van Wolveren WJ, Plomp S, et al. Inflammation of the atherosclerotic cap and shoulder of the plaque is a common and locally observed feature in unruptured plaques of femoral and coronary arteries. Arterioscler Thromb Vasc Biol 1999;19:54-58.

26. Shi MC, Wang SC, Zhou HW, Xing YQ, Cheng YH, Feng JC, et al. Compensatory remodeling in symptomatic middle cerebral artery atherosclerotic stenosis: a high-resolution MRI and microemboli monitoring study. Neurol Res 2012;34:153-158.

27. Watanabe $Y$, Nagayama M. MR plaque imaging of the carotid artery. Neuroradiology 2010;52:253-274.

28. Suzuki J, Takaku A. Cerebrovascular "moyamoya" disease. Disease showing abnormal net-like vessels in base of brain. Arch Neurol 1969;20:288-299.

29. Bang OY, Ryoo S, Kim SJ, Yoon CH, Cha J, Yeon JY, et al. Adult moyamoya disease: a burden of intracranial stenosis in East Asians? PLoS One 2015;10:e0130663.

30. Kim JS. Moyamoya disease: epidemiology, clinical features, and diagnosis. J Stroke 2016;18:2-11.

31. Kaku Y, Morioka M, Ohmori Y, Kawano T, Kai Y, Fukuoka H, et al. Outer-diameter narrowing of the internal carotid and middle cerebral arteries in moyamoya disease detected on 3D constructive interference in steady-state MR image: is arterial constrictive remodeling a major pathogenesis? Acta Neurochir (Wien) 2012;154:2151-2157.

32. Takagi Y, Kikuta K, Nozaki K, Hashimoto N. Histological features of middle cerebral arteries from patients treated for Moyamoya disease. Neurol Med Chir (Tokyo) 2007;47:1-4

33. Kim YJ, Lee DH, Kwon JY, Kang DW, Suh DC, Kim JS, et al. High resolution MRI difference between moyamoya disease and intracranial atherosclerosis. Eur J Neurol 2013;20:1311-1318.

34. Burke GM, Burke AM, Sherma AK, Hurley MC, Batjer HH, Bendok BR. Moyamoya disease: a summary. Neurosurg Focus 2009;26:E11.

35. Choi YJ, Jung SC, Lee DH. Vessel wall imaging of the intracranial and cervical carotid arteries. J Stroke 2015;17:238-255.

36. Debette S, Compter A, Labeyrie MA, Uyttenboogaart M, Metso TM, Majersik JJ, et al. Epidemiology, pathophysiology, diagnosis, and management of intracranial artery dissection. Lancet Neurol 2015;14:640-654.

37. Kwon JY, Kim NY, Suh DC, Kang DW, Kwon SU, Kim JS. Intracranial and extracranial arterial dissection presenting with ischemic stroke: lesion location and stroke mechanism. J Neurol Sci 2015;358:371-376.

38. Maruyama H, Nagoya H, Kato Y, Deguchi I, Fukuoka T, Ohe Y, et al. Spontaneous cervicocephalic arterial dissection with headache and neck pain as the only symptom. J Headache Pain 2012;13:247-253. 
39. Lee SH, Kim KY, Jung JM. High-resolution magnetic resonance imaging for the follow-up of intracranial arterial dissections. Acta Neurol Belg 2020 Jul 10. [Epub]. DOl:10.1007/s13760-020-014320 . Online ahead of print

40. Lee SH, Jung JM, Kim KY, Kim BJ. Intramural hematoma shape and acute cerebral infarction in intracranial artery dissection: a high-resolution magnetic resonance imaging study. Cerebrovasc Dis 2020;49:269-276.

41. Patel RR, Adam R, Maldjian C, Lincoln CM, Yuen A, Arneja A. Cervical carotid artery dissection: current review of diagnosis and treatment. Cardiol Rev 2012;20:145-152.

42. Wu Y, Wu F, Liu Y, Fan Z, Fisher M, Li D, et al. High-resolution magnetic resonance imaging of cervicocranial artery dissection: imaging features associated with stroke. Stroke 2019;50:3101-3107.

43. Wang Y, Lou X, Li Y, Sui B, Sun S, Li C, et al. Imaging investigation of intracranial arterial dissecting aneurysms by using $3 \mathrm{~T}$ high-resolution MRI and DSA: from the interventional neuroradiologists' view. Acta Neurochir (Wien) 2014;156:515-525.

44. Redekop GJ. Extracranial carotid and vertebral artery dissection: a review. Can J Neurol Sci 2008;35:146-152.

45. Calabrese LH, Dodick DW, Schwedt TJ, Singhal AB. Narrative review: reversible cerebral vasoconstriction syndromes. Ann Intern Med 2007;146:34-44.
46. Miller TR, Shivashankar R, Mossa-Basha M, Gandhi D. Reversible cerebral vasoconstriction syndrome, part 1: epidemiology, pathogenesis, and clinical course. AJNR Am J Neuroradiol 2015;36:1392-1399.

47. Miller TR, Shivashankar R, Mossa-Basha M, Gandhi D. Reversible cerebral vasoconstriction syndrome, part 2: diagnostic work-up, imaging evaluation, and differential diagnosis. AJNR Am J Neuroradiol 2015;36:1580-1588.

48. Swartz RH, Bhuta SS, Farb Rl, Agid R, Willinsky RA, Terbrugge $K G$, et al. Intracranial arterial wall imaging using high-resolution 3-tesla contrast-enhanced MRI. Neurology 2009;72:627-634.

49. Hajj-Ali RA, Calabrese LH. Diagnosis and classification of central nervous system vasculitis. J Autoimmun 2014;48-49:149-152.

50. Jung SC, Kang DW, Turan TN. Vessel and vessel wall imaging. Front Neurol Neurosci 2016;40:109-123.

51. Lindenholz A, van der Kolk AG, Zwanenburg JJM, Hendrikse J. The use and pitfalls of intracranial vessel wall imaging: how we do it. Radiology 2018;286:12-28.

52. Power S, Matouk C, Casaubon LK, Silver FL, Krings T, Mikulis DJ, et al. Vessel wall magnetic resonance imaging in acute ischemic stroke: effects of embolism and mechanical thrombectomy on the arterial wall. Stroke 2014;45:2330-2334. 